\title{
Title: In Silico Study of Silybum Marianum Targeting PARP protein (4UND protein)
}

\section{Authors: Hrishikesh D. GOSAVI풀. Dravin BADHE²}

${ }^{1}$ Student, Department of Pharmacy, Navsahyadri Institute of Pharmacy, Pune, INDIA.

${ }^{2}$ Associate Professor, Pharmaceutics Department, Navsahyadri Institute, of Pharmacy, Pune, INDIA.

Affiliations: Mr. Hrishikesh Gosavi; Dr. Pravin Badhe.

Corresponding Authors E-Mail-gosavihrishikesh@gmail.com

\section{Abstract-}

Silybum Marianum, is a plant belonging to the family Asteraceae. For many centuries it has been used a natural remedy for many diseases like Liver and Biliary tract diseases. It is effective as an antioxidant and is used in a variety of diseases. This study was conducted to check the effects of Silybum Marianum on PARP protein (4UND protein).

The Molecular Docking techniques was chosen to check the effects of different chemical constituents of Silybum marianum on DNA damaging protein. For this purpose, different PARP inhibitor drugs were taken as standard. The Molecular Docking of the chemical constituents of Silybum marianum was performed using 4UND protein with the help of PyRx software along with BIOVIA Drug Discovery studio software. The result of molecular docking showed that some of the chemical constituent have higher binding affinity than standard PARP inhibitor drugs.

\section{Keywords-}

Silybum Marianum, Parp protein, 4UND protein, Molecular Docking, PyRx, BIOVIA Drug Discovery.

\section{Introduction-}

Milk thistle which is also known as Silybum Marianum, belongs to the family of $\underline{\text { Asteraceae }}$ and is an annual or biennial herbaceous plant local of the Mediterranean basin, it is these days widely spread in Central Europe, America and South-Australia [1][2]. It can also be found in India, China, and Africa [3]. There are two species of Genus Silybum(L). Gaertn - $\underline{S}$.Marianum with variegated leaves and $\underline{S}$. Eburneum Coss \& Dureu with completely green leaves [4]. It grows to a peak of $60-150 \mathrm{~cm}$ 
and it has white-veined leaves with toothed spiny edges and purple flowers on the pinnacle of the stem and the ramifications ripe into 6-7 $\mathrm{mm}$ long brownish fruits (achenes) with a white silky pappus at their apex and has big prickly edged leaves included with undulating white patches and stems containing a milky juice [5][6][7].
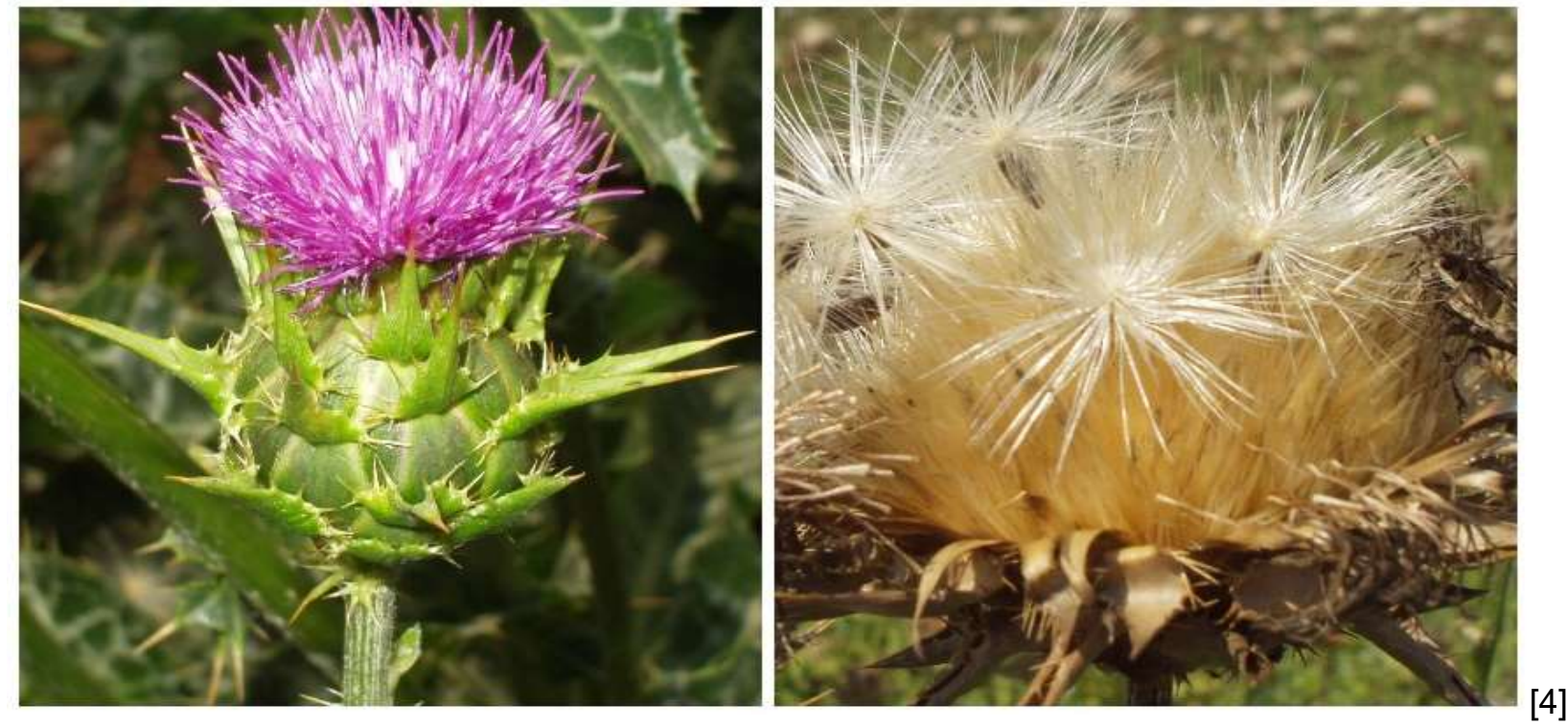

Fig. Silybum Marianum

Milk Thistle's active component is a lipophilic extract obtained from the seeds of the plant, and it contains three isomer flavonolignans (silybin, silydianin, and silychristin), together known as silymarin [8][9].

Because of the existence of phytoconstituents such as antioxidants and complete phenolics, milk thistle is used as a medicine for the treatment of a variety of diseases [9]. For years, Silybum Marianum has been used as a natural treatment for liver and biliary tract diseases [10]. A study that was done was to see how effective Silybum Marianum is at scavenging free radicals in vitro and how powerful it is as an antioxidant [11]. Milk thistle extracts are currently being researched for chemoprevention, recovery, and amelioration of chemotherapy side effects in cancer experimental therapeutics, but however, nomenclature precision has fallen behind [12]. Preclinical evidence for silymarin`s hepatoprotective and anti-carcinogenic effects, includes cancer cell growth inhibition in humans, prostate, skin, breast and cervical cells [13]. Extracts from milk thistle seeds have shown efficacy in halting the progression of human prostate carcinoma in a variety of in vitro and in vivo preclinical 
models over the last few years [14]. It has also been proposed that Milk Thistle can be used in diabetes to diminish insulin resistance [15].

$\mathrm{NAD}+$ protein (ADP-ribosyl) transferase (polymerizing) (PARP) is a widely distributed chromatinassociated protein that is highly conserved and found in all eukaryotes except yeast [16]. PARP attaches to DNA strand, breaks and catalyses the transfer of ADP ribose from its substrate NAD+ to a number of nuclear acceptors, mostly to itself, in response to DNA damage induced by external genotoxic chemicals and endogenous cellular processes [17]. PARP-1 uses its two zinc fingers to detect DNA nicks, which it catalytically activates by binding to the break and helps to repair. The only other PARP enzyme activated by and assisting in the repair of DNA breaks is PARP-2, which has the closest structural resemblance to PARP-1 [18][19]. Of all the proven use of PARP inhibitors the most promising ones are ischemia and cancer [20]. So, we have decided to do the research on cancer.

While doing this research we have identified the research gap that no one else has used 4UND protein before for Molecular Docking of chemical constituents of Silybum Marianum on anti-cancer activity so, we have used 4UND protein.

Based on the above information, we intend to carry out in silico study of chemical constituents of Silybum Marianum with PARP protein. We are doing this study so that we can check the binding affinity of chemical constituents of Silybum Marianum and compare it with the binding affinity of present PARP inhibitor drugs i.e., Talazoparib, Rucaparib, Olaparib, Niraparib and if the results acquired are good, we can create new drugs which can show better anti-cancer activity than present drugs which are available.

The purpose of this study was to know about the docking affinity of the chemical constituents of Silybum Marianum. The Molecular Docking was done by using the PyRx software and BIOVIA Drug Discovery. The necessary proteins and ligands were downloaded from protein bank and PubChem respectively.

Botanics-

Botanical Name- Silybum Marianum

Kingdom- Plantae 
Division- Magnoliophyta

Class- Magnoliopsida

Subclass- Asteridae

Order- Asterales

Family- Asteraceae

Genus- Silybum

Species- Silybum Marianum [21]

\section{Chemical Constituents of Silybum Marianum-}

Oil content $26.05 \%$, moisture $4.48 \%$, ash $1.93 \%$, crude fibre $5.48 \%$, carbs $87.2 \%$, and total proteins 23\% make up Silybum Marianum seeds. Apigenin, silybonol, proteins, betaine, fixed oil, and free fatty acids are active elements found in the seeds of the milk thistle. It also contains sugars such asarabinose, rhamnose, xylose, glucose [4][9].

The main bioactive component of medicinal plant (milk thistle) is silymarin (4\% to $6 \%$ ). Silymarin is the mixture of different flavonolignans such as, silybinin (60\%) $A$ and $B$ (SBN $A \& B$ ), iso-silybinin $A$ and $B$ (ISBN A\&B), silychristin (SCN), and silydianin (SDN), it is a well-known Chinese herb. Silymarin is also present in the seeds, fruits as well as in the leaves of the Milk Thistle but the seed part has the maximum concentration of silymarin. Besides this Milk Thistle also includes other flavonoids such asRutin, quercetin, dihydrokaempferol, kaempferol, apigenin, naringin, eriodyctiol, chrysoeriol [4][9][22].

Other constituents are fixed oil (16\% to $18 \%$ ), betaine, trimethyl-glycine and amines, $20 \%-30 \%$ fixed oil, of which approximately $60 \%$ is linoleic acid, glutamic acid, approximately $30 \%$ is oleic acid, and approximately $9 \%$ palmitic acid, $25-30 \%$ protein, $0.038 \%$ tocopherol, $0.63 \%$ sterols- including cholesterol, campesterol, stigmasterol, and sitosterol, some mucilage, 2,2-diphenyl-1- picrylhydrazyl, [22]. The fatty acids that are present are saturated fatty acids- Lauric (C12:0), Myristic (C 14:0), Palmitic (C 16:0), Stearic (C 18:0), Arachidic (C 20:0), Behenic (C 22:0), Lignoceric (C 24:0), Palmitoleic (C 16:1), Heptadecenoic (C 17:1), Oleic (C 18:1), Vaccenic (C 18:1), Eicosenoic (C 20:1), Erucic (C 22:1). 
Polyunsaturated fatty acids are- Linoleic, Linolenic. The ash content is $9.6 \%$ in stalks, $10.5 \%$ in leaves and $9.7 \%$ in heads [23].

The essential amino acids present in Silybum Marianum are- Isoleucine, Leucine, Lysine, Methionine, Phenylalanine, Threonine, Valine, Histidine, and Non-Essential amino acids present are- Alanine, Aspartic acid, Glutamic acid, Glycine, Proline, Serine, Arginine, Cysteine, Tyrosine [23].

The mineral content of Silybum Marianum is-

- $\mathrm{Na}-81.0$

- K-718.0

- Ca - 132.0

- $\mathrm{Mg}-17.3$

- $\mathrm{Cu}-0.08$

- $\mathrm{Fe}-0.50$

- $\quad M n-0.10$

- $\quad \mathrm{Zn}-0.26$ [23].

\section{Molecular Docking-}

The field of molecular docking has grown in popularity over the last three decades as a result of the structural molecular biology and structure-based drug discovery requirements [24]. Molecular docking is a type of computational modelling that helps predict the preferred binding orientation of one molecule (e.g., ligand) to another (e.g., receptor) when they interact to form a stable complex [25].

\section{Materials-}

Hardware \& Software- HP 15-da0300TU with Intel core CPU of $2.57 \mathrm{Ghz}, 8 \mathrm{gb}$ ram, and intel integrated graphics card of $4 \mathrm{gb}$, and 64-bit windows operating system.

PyRx a software [26] written in python language, which today can be used on any PC (personal computer), is used along with BIOVIA Drug Discovery software [27] for molecular docking. 
The files used for molecular docking were ligands which were downloaded from PubChem [28] in "sdf" format and Proteins which were downloaded from Protein bank in "pdb" format [29].

\section{Methods-}

The protein files that were downloaded were first converted to "pdbqt" format from "pdb" format. The conversion of file can be done in the PyRx software which includes Auto Dock Vina.

1- Start by double clicking on PyRx icon on your desktop.

2- Select file option on the right-hand side corner of your screen, and then choose the "Load Molecule" option and select your protein.

3- Then right click on the selected protein and choose the Auto Dock option and select the "Make Macromolecule" option.

4- Then select the "Open Babel" option on the lower side of your screen. Then click on the right side first icon of insert new item, then select the ligands one by one.

5- Then right click on the selected ligand and choose the Minimize All option.

6- After that right click again and select the "convert all to AutoDock Ligand(pdbqt) option.

7- Then select the vina wizard and click on start.

8- Select the ligands using cntrl button on your keyboard and and using cursor.

9- Then on the screen the structure will appear make sure the whole structure is in the box.

10-Then click on Forward button and the docking will start.

11-After completion of the docking process click on "save as comma-separated vale" on the right side of your screen, and the binding affinity results will be saved in excel sheet. 


\section{$\underline{\text { Results- }}$}

The results that are given below shows the binding affinity between Standard PARP inhibitor drugs i.e., Talazoparib, Rucaparib, Olaparib, and Niraparib with PARP protein (4UND protein) and Chemical constituents of Silybum Marianum i.e., Silychristin, Silydianin, and Naringin with PARP protein (4UND protein).

Table1 shows the binding affinity of standard PARP inhibitor drugs with 4UND PARP protein and Table 2 shows the binding affinity of chemical constituents of Silybum Marianum with 4UND PARP protein.

\section{A) Standard}

Table-1.

\begin{tabular}{|l|l|l|l|}
\hline Sr.No & Complex (Protein + Ligand) & Ligands & $\begin{array}{l}\text { Binding affinity } \\
\text { (kcal/mol) }\end{array}$ \\
\hline 1 & 4und_(1)_135565082_uff_E=560.92 & Talazoparib & -8.9 \\
\hline 2 & 4und_(1)_9931954_uff_E=653.31 & Rucaparib & -7.8 \\
\hline 3 & 4und_(1)_23725625_uff_E=1707.13 & Olaparib & -9.8 \\
\hline 4 & 4und_(1)_24958200_uff_E=461.77 & Niraparib & -8.2 \\
\hline
\end{tabular}

B) Chemical Constituents

\begin{tabular}{|l|l|l|l|}
\hline Sr.No & Complex (protein + Ligand) & Ligands & $\begin{array}{l}\text { Binding Affinity } \\
\text { (kcal/mol) }\end{array}$ \\
\hline 1 & 4und_(1)_441764_uff_E=498.05 & Silychristin & -10.1 \\
\hline 2 & 4und_(1)_11982272_uff_E=629.25 & Silydianin & -11.2 \\
\hline 3 & 4und_(1)_442428_uff_E=615.92 & Naringin & -11.2 \\
\hline
\end{tabular}

Table- 2.

The binding affinity of the other chemical constituents of Silybum Marianum which are also found to be good but not better than the standard PARP inhibitor drugs has been shown in Table 3 in appendix section as well as the binding affinity of the chemical constituents of Silybum Marianum whose binding affinity was found to be less than -6 is shown in Table 4 in appendix. 


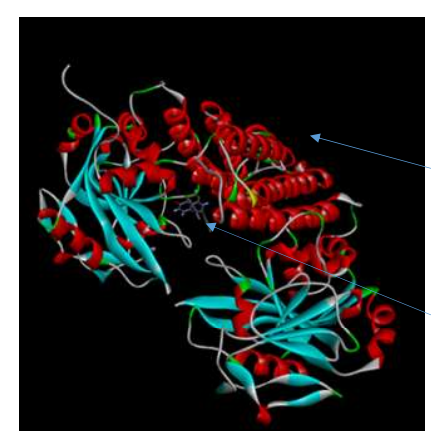

Talazoparib

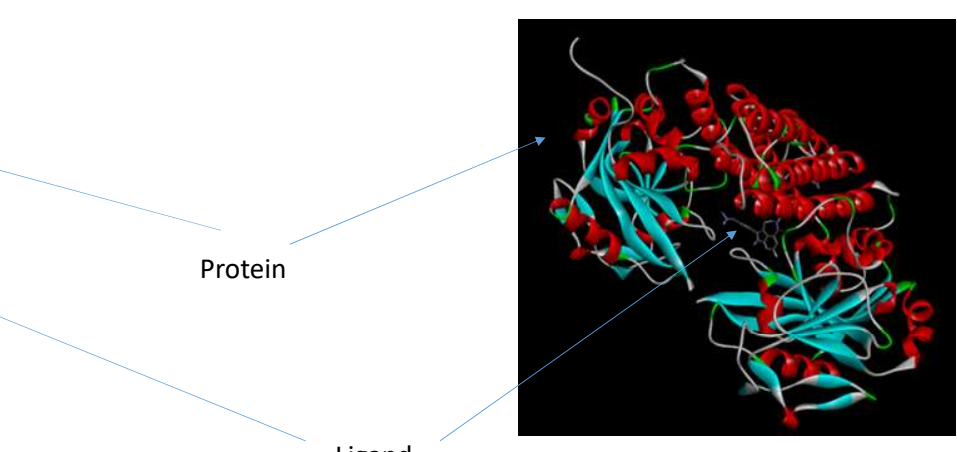

Rucaparib

Fig. Binding of Standard PARP inhibitor drugs with 4UND protein

Figure- 1.

Figure 1 shows the binding between Standard PARP inhibitors i.e., Talazoparib and Rucaparib with 4UND protein (Table-1). The binding affinity of Talazoparib with 4UND protein was found to be -8.9 $\mathrm{kcal} / \mathrm{mol}$ and the binding affinity of Rucaparib with 4UND protein was found to be $-7.8 \mathrm{kcal} / \mathrm{mol}$. The figure shows the protein and the ligand marked with arrows. The Red, blue, yellow and green colour section of the figure shows the protein i.e., 4UND PARP protein and the violet colour section shows the ligands i.e., Talazoparib and Rucaparib. 


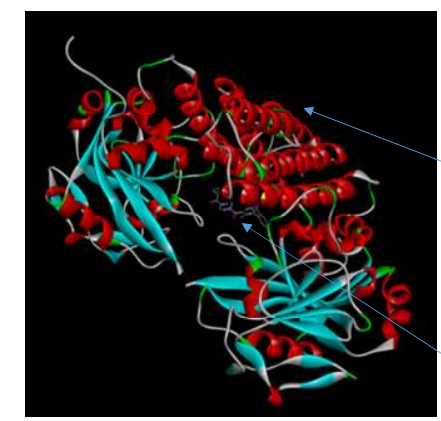

Olaparib

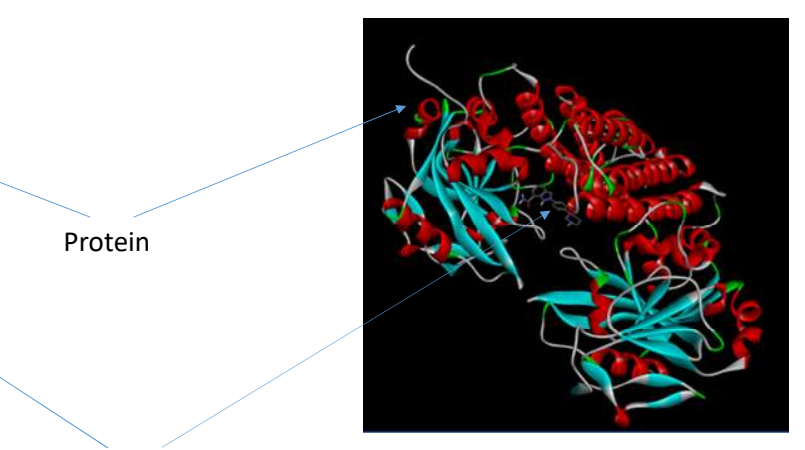

Ligand

Niraparib

Fig. Binding of Standard PARP inhibitor drugs with 4UND protein

Figure- 2.

Figure 2 shows the binding between Standard PARP inhibitors i.e., Olaparib and Niraparib with 4UND protein (Table- 1). The binding affinity of Olaparib with 4UND protein was found to be $-9.8 \mathrm{kcal} / \mathrm{mol}$ and the binding affinity of Niraparib with 4UND protein was found to be $-8.2 \mathrm{kcal} / \mathrm{mol}$. The figure shows the protein and the ligand marked with arrows. The Red, blue, yellow and green colour section of the figure shows the protein i.e., 4UND PARP protein and the violet colour section shows the ligands i.e., Olaparib and Niraparib. 


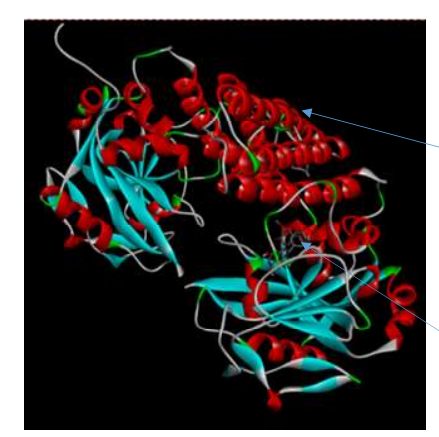

Silydianin

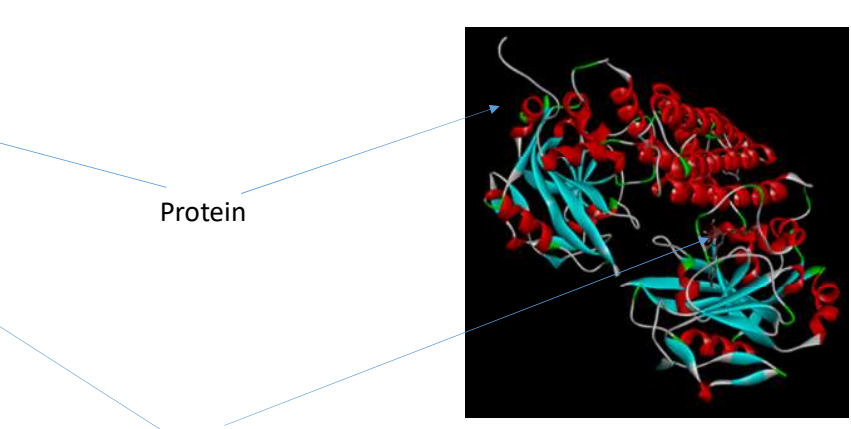

Ligand

Naringin

Fig. Binding of Chemical Constituents of Slybum Marianum with 4UND protein (This figure shows chemical constituents of Silybum Marianum with best binding affinity with 4UND protein)

Figure- 3.

Figure 3 shows the binding between Chemical constituents of Silybum Marianum i.e., Silydianin and Naringin with 4UND protein (Table- 2). The binding affinity of Silydianin with 4UND protein was found to be $-11.2 \mathrm{kcal} / \mathrm{mol}$ and the binding affinity of Naringin with 4UND protein was found to be -11.2 $\mathrm{kcal} / \mathrm{mol}$. The binding affinity of Silydianin and Naringin with 4UND protein was found to be better than the binding affinity of standard PARP inhibitor drugs with 4UND protein. The figure shows the protein and the ligand marked with arrows. The Red, blue, yellow and green colour section of the figure shows the protein i.e., 4UND PARP protein and the violet colour section shows the ligands i.e., Silydianin and Naringin. 


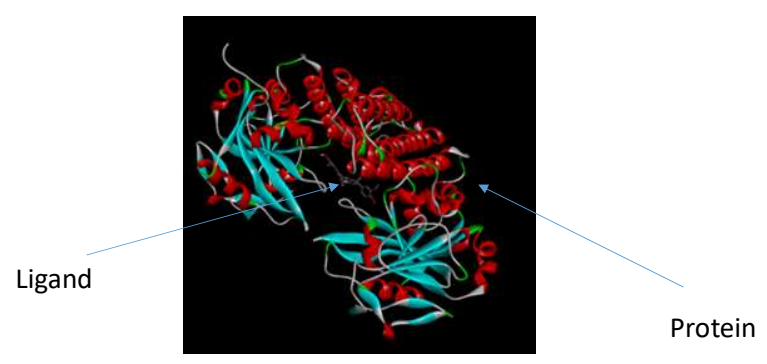

Silychristin

Fig. Binding of Chemical Constituents of Slybum Marianum with 4UND protein (This figure shows chemical constituents of Silybum Marianum with best binding affinity with 4UND protein)

\section{Figure- 4.}

Figure 4 shows the binding between the Chemical Constituent of Silybum Marianum i.e., Silychristin with 4UND protein (Table-2). The binding affinity of Silychristin with 4UND protein was found to be $10.1 \mathrm{kcal} / \mathrm{mol}$. The binding affinity of Silychristin with 4UND protein was found to be better than the binding affinity of standard PARP inhibitor drugs with 4UND protein. The figure shows the protein and the ligand marked with arrows. The Red, blue, yellow and green colour section of the figure shows the protein i.e., 4UND PARP protein and the violet colour section shows the ligands i.e., Silychristin.

The remaining images of binding of chemical constituents of Silybum Marianum with 4UND protein are added in the Appendix section.

From the above results it can be said that, Silydianin, Naringin, and Silychristin which are the chemical constituents of Silybum Marianum shows better binding affinity than Talazoparib, Rucaparib, Olaparib, and Niraparib. Talazoparib, Rucaparib, Olaparib, and Niraparib all are Standard PARP inhibitor drugs.

Silymarin, Silybinin, Apigenin, Quercetin and Campesterol all are the chemical constituents of Silybum Marianum and shows better binding affinity than Talazoparib. Silymarin, Silybinin, Apigenin, Quercetin, 
Campesterol, and Sitosterol, shows better binding affinity than Rucaparib. Silymarin, Silybinin, Apigenin, Quercetin, Campesterol and Sitosterol, shows better binding affinity than Niraparib.

\section{$\underline{\text { Discussion- }}$}

For centuries, Silybum Marianum has been used in the treatment of various diseases mainly liver and biliary tract related diseases. It has been used as a natural remedy for all these centuries. In this study carried out by using Molecular Docking the binding affinity of chemical constituents of Silybum Marianum with 4UND PARP protein was carried out. The binding affinity of Silydianin and Naringin which are the chemical constituents of Silybum Marianum showed good interaction with 4UND protein.

The binding affinity was found out to be as followed:

The binding affinity of Standard PARP inhibitor drugs was found to be-

1- Talazoparib: $-8.9 \mathrm{kcal} / \mathrm{mol}$.

2- Rucaparib: $-7.8 \mathrm{kcal} / \mathrm{mol}$.

3- Olaparib: $-9.8 \mathrm{kcal} / \mathrm{mol}$.

4- Niraparib: $-8.2 \mathrm{kcal} / \mathrm{mol}$.

As we can see the best binding affinity among Standard PARP inhibitor drugs was found to be of OLAPARIB which is $-9.8 \mathrm{kcal} / \mathrm{mol}$.

The binding affinity of chemical constituents of Silybum Marianum was found to be as followed:

1- Silymarin: $-9.4 \mathrm{kcal} / \mathrm{mol}$.

2- Silybinin: $-9.3 \mathrm{kcal} / \mathrm{mol}$.

3- Silychristin: $-10.1 \mathrm{kcal} / \mathrm{mol}$.

4- Silydianin: $-11.2 \mathrm{kcal} / \mathrm{mol}$.

5- Apigenin: $-9.2 \mathrm{kcal} / \mathrm{mol}$.

6- Quercetin: $-9.3 \mathrm{kcal} / \mathrm{mol}$.

7- Dihydrokaempferol: $-7.2 \mathrm{kcal} / \mathrm{mol}$.

8- Kaempferol: $-7.4 \mathrm{kcal} / \mathrm{mol}$.

9- Naringin: $-11.2 \mathrm{kcal} / \mathrm{mol}$.

10-Eriodycitol: $-7.8 \mathrm{kcal} / \mathrm{mol}$. 
11-Chrysoeriol: $-7.6 \mathrm{kcal} / \mathrm{mol}$.

12-Campesterol: $-9.1 \mathrm{kcal} / \mathrm{mol}$.

13-Sitosterol: $-8.8 \mathrm{kcal} / \mathrm{mol}$.

14- Rutin: $-8.9 \mathrm{kcal} / \mathrm{mol}$.

As we can see the binding affinity of Silydianin which is found to be $-11.2 \mathrm{kcal} / \mathrm{mol}$ and the binding affinity of Naringin which was also found to be $-11.2 \mathrm{kcal} / \mathrm{mol}$ which is the best amongst all the chemical constituents of Silybum Marianum. From this we can say that the binding affinity of Silydianin and Naringin was found to be better than the binding affinity of Olaparib. And also, the binding affinity of Silychristin which is $-10.1 \mathrm{kcal} / \mathrm{mol}$ which is also better than Olaparib, Niraparib, Rucaparib and Talazoparib.

The previous proven activities of Silydianin, Naringin and Silychristin are- Silydianin which is an active chemical constituent of Silybum Marianum is proven to have inhibitory effect [30], antioxidant and cytotoxic activities in Caco-2 cells [31], used in prostate cancer [32], PTP1B inhibitor [33]. Naringin which is also a chemical constituent of Silybum Marianum shows antioxidant, anti-inflammatory, antiapoptotic, anti-ulcer, anti-osteoporotic and anti-carcinogenic activity [34], Anti-bacterial [35], it regulates p21 and survivin in triple negative breast cancer cells [36]. Silychristin which is also a chemical constituent of Silybum Marianum shows anti-oxidant, anti-inflammatory and Multidrug Resistance Modulation Activity [37], used in cancer prevention [38]. So, from these proven activities we can say that Silydianin, Naringin and Silychristin are previously being used for their anti-cancer activity and from the results of molecular docking we can also say that they can be used as PARP inhibitors in the future.

The binding affinity of Silymarin $-9.4 \mathrm{kcal} / \mathrm{mol}$, Silybinin $-9.3 \mathrm{kcal} / \mathrm{mol}$, Apigenin $-9.2 \mathrm{kcal} / \mathrm{mol}$, Quercetin $-9.3 \mathrm{kcal} / \mathrm{mol}$ and Campesterol $-9.1 \mathrm{kcal} / \mathrm{mol}$ was found to be better than Talazoparib $-8.9 \mathrm{kcal} / \mathrm{mol}$. The binding affinity of Rutin $-8.9 \mathrm{kcal} / \mathrm{mol}$ was found to be equal to Talazoparib.

The binding affinity of Sitosterol $-8.8 \mathrm{kcal} / \mathrm{mol}$ was to be better than Rucaparib which is $-7.8 \mathrm{kcal} / \mathrm{mol}$. The binding affinity of Eriodycitol $-7.8 \mathrm{kcal} / \mathrm{mol}$ was found to be equal to Rucaparib. The binding affinity of Silymarin $-9.4 \mathrm{kcal} / \mathrm{mol}$, Silybinin $-9.3 \mathrm{kcal} / \mathrm{mol}$, Apigenin $-9.2 \mathrm{kcal} / \mathrm{mol}$, Quercetin $-9.3 \mathrm{kcal} / \mathrm{mol}$, Campesterol $-9.1 \mathrm{kcal} / \mathrm{mol}$, Sitosterol $-8.8 \mathrm{kcal} / \mathrm{mol}$ and Rutin $-8.9 \mathrm{kcal} / \mathrm{mol}$ was found to be better than Niraparib $-8.2 \mathrm{kcal} / \mathrm{mol}$.

PARP inhibitors are a cancer therapy targeting poly (ADP-ribose) polymerase were the first drugs to get approval to exploit synthetic lethality. The standard PARP inhibitor Drugs used were Talazoparib, Rucaparib, Olaparib, and Niraparib [39]. 
Olaparib was the first PARP inhibitor drug to get approved by European Medicines Agency in 2014, for mutant ovarin cancer [40] it also shows effect in late chemotherapy-refractory BRCA-deficient breast cancer and also olaparib is well tolerated and very active [41]. Rucaparib a PARP inhibitor drug is known to be tolerable and shows activity in patients with platinum-sensitive germline BRCA $1 / 2$ mutated-HGOC [42]. Talazoparib which is a PARP inhibitor drug inhibits PARP catalytic activity, trapping PARP1 on damaged DNA and causing cell death in BRCA 1/2 -mutated cells [43]. Niraparib a potent PARP $1 / 2$ inhibitor drug with good cell activity shows promising activity in cancer patients [44].

So, on the basis of the result obtained we can expect that Silydianin and Naringin also possess the inhibition activity against Oxidative DNA damage, because Silybum marianum extract has the ability to show protection against photo-carcinogenesis [45] and also on the basis of the results of molecular docking we can expect that in the future Silybum Marianum can be used to create PARP inhibitor drugs.

\section{$\underline{\text { Conclusion- }}$}

The analysis of the results of Molecular Docking of chemical constituents of Silybum Marianum with 4UND DNA damaging protein helped to examine and evaluate the binding affinity of chemical constituents of Silybum Marianum with 4UND DNA damaging protein. The results obtained showed that Silydianin and Naringin the chemical constituents of Silybum Marianum showed the binding affinity of $-11.2 \mathrm{kcal} / \mathrm{mol}$ respectively, which is better than the best binding affinity amongst the Standard PARP inhibitor drugs which was shown by Olaparib which is $-9.8 \mathrm{kcal} / \mathrm{mol}$.

From the above result we can conclude that Silydianin and Naringin, the chemical constituents of Silybum Marianum showed better binding affinity than Talazoparib, Rucaparib, Olaparib, and Niraparib which are the standard PARP inhibitor drugs. Also, Silychristin which is a chemical constituent of Silybum Marianum shows the binding affinity of $-10.1 \mathrm{kcal} / \mathrm{mol}$, which is also better than Talazoparib, Rucaparib, Olaparib, and Niraparib. So, we can say that in the future we can expect to use Silybum Marianum to create PARP inhibitor drugs.

\section{References-}

1- F. Gresta, Avola, G., \& Guarnaccia, P. Agronomic Characterization of Some Spontaneous Genotypes of Milk Thistle (Silybum marianum L. Gaertn) in Mediterranean Environment. Journal of Herbs, Spices \& Medicinal Plants, 12(4), 51-60, 2006. 
2- Singh, R. P., \& Agarwal, R. "Mechanisms and preclinical efficacy of silibinin in preventing skin cancer" European Journal of Cancer, 41(13), 1969-1979, 2005.

3- M.R.Rady, M.M.Saker, M.A.Matter, “In vitro culture, transformation and genetic fidelity of Milk Thistle" journal of genetic engineering and biotechnology, vol 16, issue 2, 2018.

4- Veronica Valkova, Hana Duranova, Jana Bilcikova, Miroslav Haban, "Milk Thistle (Silybum Marianum): A valuable Medicinal Plant with Several Therapeutic Purposes" journal of Microbiology, Biotechnology and Food Sciences, vol 9, no 4, 2020.

5- Javad Roustakhiz and Ebrahim Saboki, "Plant Tissue Culture of Silybum Marianum L. and Check out Elicitor Effect on the Amount of Silymarin” Journal of Novel Applied Sciences, 2016.

6- Hadolin, M., Skerget, M., Knez, Z., \& Bauman, D. High pressure extraction of vitamin E-rich oil from Silybum marianum. Food Chemistry, 74(3), 355-364, 2001.

7- David E. Zaurov, Igor V. Belolipov, Anvar G. Kurmukov, Ishenbay S. Sodombekov, Anarbek A. Akimaliev, Sasha W. Eisenman "The Medicinal Plants of Uzbekistan and Kyrgyzstan”, 2012.

8- Ludovico Abenavoli, Raffaele Capasso, Natasa Milic, Francesco Capasso, "Milk thistle in liver diseases: past, present, future" 2010.

9- Maryam Aziz, Farhan Saeed, Nazir Ahmad, Aftab Ahmad, Muhammad Afzaal, Shahzad Hussain, Abdellatif A. Mohamed, Mohamed S. Alamri, Faqir Muhammad Anjum, "Biochemical profile of milk thistle (Silybum Marianum L.) with special reference to silymarin content" 2020.

10- Flora, K. "Milk Thistle (Silybum marianum) for the Therapy of Liver Disease" The American Journal of Gastroenterology, 93(2), 139-143, 1998.

11-Zarban Asghar and Ziaee Masood, "Evaluation Of Antioxidant Properties Of Silymarin And Its Potential To Inhibit Peroxyl Radicals In Vitro" Department of Biochemistry, Birjand University of Medical Sciences, Birjand, Iran, Department of Internal Medicine, Birjand University of Medical Sciences, Birjand, Iran 2008.

12-Kroll, D. J., Shaw, H. S., \& Oberlies, N. H. “Milk Thistle Nomenclature: Why It Matters in Cancer Research and Pharmacokinetic Studies” Integrative Cancer Therapies, 6(2), 110-119, 2007.

13-Post-White, J., Ladas, E. J., \& Kelly, K. M. (2007). Advances in the Use of Milk Thistle (Silybum marianum). Integrative Cancer Therapies, 6(2), 104-109.

14-Davis-Searles, P. R., Nakanishi, Y., Kim, N.-C., Graf, T. N., Oberlies, N. H., Wani, M. C., Kroll, D. J. "Milk Thistle and Prostate Cancer: Differential Effects of Pure Flavonolignans fromSilybum 
marianumon Antiproliferative End Points in Human Prostate Carcinoma Cells" Cancer Research, 65(10), 4448-4457, 2005.

15-Shane-McWhorter, L. Biological Complementary Therapies: A Focus on Botanical Products in Diabetes. Diabetes Spectrum, 14(4), 199-208, 2001.

16- Z. Q. Wang, B. Auer, L .Stingl, H. Berghammer, D. Haidacher, M. Schweiger, and E. F. Wagner, "Mice lacking ADPRT and poly(ADP-ribosyl)ation develop normally but are susceptible to skin disease" Research Institute of Molecular Pathology (IMP), Vienna, Austria, 1995.

17-Wang, Z.-Q., Stingl, L., Morrison, C., Jantsch, M., Los, M., Schulze-Osthoff, K., \& Wagner, E. F. (1997). PARP is important for genomic stability but dispensable in apoptosis. Genes \& Development, 11(18), 2347-2358.

18-Zaremba, T., \& Curtin, N. (2007). PARP Inhibitor Development for Systemic Cancer Targeting. Anti-Cancer Agents in Medicinal Chemistry, 7(5), 515-523.

19-Schreiber, V. Ame, J-C, Dolle, P., Schultz I., RinaldiB., Fraulob V., Menissier-de Murcia J., de Murcia G. J., Biol. Chem., 2002, 277, 23028.

20- Dana V. Ferraris "Evolution of poly (ADP ribose) Polymerase -1 (PARP-1) Inhibitors from concept to Clinic" John Hopkins University Brain Science Institute, 2010.

21-Blessed Milk Thistle Silybum Marianum (L.) Gaertn, The University Of Georgia Centre for invasive species Ecosystem health, 2018.

22- Ajit Kiran Kaur, A.K.Wahi, Brijesh kumar, Anil Bhandari, Neelkant Prasad, "International Journal Of Pharma. Research and Development" Deptt. of Pharmacy, Jodhpur National University, Jodhpur, Gyani Inder Singh Institute of Professional Studies, Dehradun, 2001.

23-Andrzejewska, J., Martinelli, T., \& Sadowska, K. "Silybum marianum: non-medical exploitation of the species" Annals of Applied Biology, 167(3), 285-297, 2015.

24-Morris, G. M., \& Lim-Wilby, M. “Molecular Docking. Molecular Modeling of Proteins”, 365-382, 2008.

25- Shweta Agarwal And Ranjana Mehrotra, “An Overview Of Molecular Docking” Quantum Optics And Photon Physics, CSIR-National Physical Laboratory, India, 2016.

26-pyRx - Virtual Screening Tool download | https://sourceforge.net/projects/pyrx/ [ last accessed on 20-05-2021]. 
27- BIOVIA Discovery Studio- https://discover.3ds.com/discovery-studio-visualizer-download [last accessed on 20-05-2021].

28- https://pubchem.ncbi.nlm.nih.gov/ [last accessed on 20-05-2021].

29- https://www.rcsb.org/ [last accessed on 20-05-2021].

30- Malgorzata Zielinska-Przyjemska and Krzysztof Wiktorowicz "An in vitro study of the protective effect of the flavonoid silydianin against reactive oxygen species" vol 20 , issue $2,2006$.

31-Naila Ben, Rahal Francisco, J Barba, Danielle Barth, Isabelle Chevalot, "Supercritical $\mathrm{CO}_{2}$ extraction of oil, fatty acids and flavonolignans from milk thistle seeds: Evaluation of their antioxidant and cytotoxic activities in Caco-2 cells" Food and Chemical Toxicology, Vol. 83, 2015.

32- Gagan Deep, Nicholas, H. Oberlies, David J. Kroll, Rajesh Agarwal, "Identifying the differential effects of silymarin constituents on cell growth and cell cycle regulatory molecules in human prostate cancer cells" International Journal of Cancer, vol. 123, issue $1,2008$.

33- Ningbo Qin, Tatsunori Sasaki, Wei Li, Jian Wang, Xiangyu Zhang, Dahong Li, Zhanli Li, Maosheng Cheng, Huiming Hua, Kazuo Koike, "Identification of flavonolignans from Silybum marianum seeds as allosteric protein tyrosine phosphatase 1B inhibitors" Journal of Enzyme inhibition and Medicinal Chemistry, vol. 33, issue 1, 2018.

34- Wang DM, Yang YJ, Zhang L, Zhang X, Guan FF, Zhang LF. "Naringin enhances CaMKII activity and improves long-term memory in a mouse model of Alzheimer's disease" Int $J$ Mol Sci. 14:5576-5586, 2013.

35- G. Celiz, M. Daz, M.C. Audisio, "Antibacterial activity of naringin derivatives against pathogenic strains" Journal of Applied Microbiology, vol. 111, issue 3, 2011.

36- Hongzhong Li, Bing Yang, Jing Huang, Tingxiu Xiang, Xuedong Yin, Jingyuan Wan, Fuling Lou, LiZhang, Hongyuan Li, Guosheng Ren, "Naringin inhibits growth potential of human triple-negative breast cancer cells by targeting $\beta$-catenin signaling pathway" Toxology letters vol. 223, issue 3, 2013.

37- Jitka Viktorova, Simona Dobiasova, Katerina Rehorova, David Biedermann, Kristyna Kanova, Karolina Seborova, Radka Vaclavikova, Katerina Valentova, Tomas Ruml, Vladimir Kren, Tomas Macek, "Antioxidant, Anti-Inflammatory, and Multidrug Resistance Modulation Activity of Silychristin Derivatives” 2019. 
38- An-Shen Lin, Makio Shibano, Kyoko Nakagwa-Goto, Harukuni Tokuda, Hideji Itokawa, Susan L. Morris-Natschke, and Kuo-Hsiung lee, "Cancer Preventive Agents. 7. AntitumorPromoting Effects of Seven Active Flavonolignans from Milk Thistle (Silybum marianum.) on Epstein-Barr Virus Activation” Pharmaceutical Biology, vol. 45, issue 10, 2007.

39-Lord, C. J., \& Ashworth, A. (2017). PARP inhibitors: Synthetic lethality in the clinic. Science, 355(6330), 1152-1158.

40- Jessica S Brown, Stan B Kaye and Timothy A Yap, "PARP inhibitors: the race is on" British Journal of Cancer, 2016.

41-A. Tutt, M. Robson, J.E. Garber, S. Domchek, M.W. Audeh, J.N. Weitz, Im. Friedlander, J. Carichael, "Phase II trial of the oral PARP inhibitor olaparib in BRCA-deficient advanced breast cancer" Jornal of Clinical oncology, Vol. 27, issue 18.

42- Rebecca Kristeleit, Geoffrey I. Shapiro, Howard A. Burris, Amit M. Oza, Patricia LoRusso, Manish R. Patel, SusanM. Domchek, Judith Balmaña, Yvette Drew, Leemay Chen, Tamar Safra, Ana Mo ntes, Heidi Giordano, Lara Maloney, Sandra Goble, Jeff Isaacson, Jim Xiao, Jen Borrow, Lindsey Rolfe and Ronnie Shapira-Frommer, "A Phase I-II Study of the Oral PARP Inhibitor Rucaparib in Patients with Germline BRCA1/2-Mutated Ovarian Carcinoma or Other Solid Tumors" Clinical Cancer research, 2017.

43- Johann Bono, RameshK. Ramanathan, Lida Mina, Rashmi Chugh, John Glaspy, Saeed Rafii, Sta n Kaye, Jasgit Sachdev, John Heymach, DavidC. Smith, JoshuaW. Henshaw, Ashleigh Herriott, Miranda Patterson, Nicola J. Curtin, Lauren Averett Byers and Zev A. Wainberg, "Phase I, DoseEscalation, Two-Part Trial of the PARP Inhibitor Talazoparib in Patients with Advanced Germline BRCA1/2 Mutations and Selected Sporadic Cancers" Cancer Discovery, 2017.

44-Philip Jones, Keith Wilcoxen, Michael Rowley and Carlo Taniatti, "Niraparib: A Poly (ADP-ribose) Polymerase (PARP) Inhibitor for the Treatment of Tumors with Defective Homologous Recombination" American Chemical Society, 2015.

45- Mudit Vaid and Santosh K. Katiyar, "Molecular mechanism of inhibition of photocarcinogenesis by silymarin a phytochemical from milk thistle (Silybum Marianum L. Gaertn) (Review), International Journal of oncology, 2010. 
Appendix-

\begin{tabular}{|l|l|l|l|}
\hline Sr.No & Complex (protein + ligand) & Ligands & $\begin{array}{l}\text { Binding } \\
\text { Affinity } \\
\text { (kcal/mol) }\end{array}$ \\
\hline 1 & 4und_(1)_5213_uff_E=444.88 & Silymarin & -9.4 \\
\hline 2 & 4und_(1)_31553_uff_E=443.51 & Silybinin & -9.3 \\
\hline 3 & 4und_(1)_5280443_uff_E=233.26 & Apigenin & -9.2 \\
\hline 4 & 4und_(1)_5280343_uff_E=380.43 & Quercetin & -9.3 \\
\hline 5 & 4und_(1)_122850_uff_E=233.92 & Dihydrokaempferol & -7.2 \\
\hline
\end{tabular}




\begin{tabular}{|l|l|l|l|}
\hline 6 & 4und_(1)_5280863_uff_E=362.50 & Kaempferol & -7.4 \\
\hline 7 & 4und_(1)_373261_uff_E=224.97 & Eriodycitol & -7.8 \\
\hline 8 & 4und_(1)_5280666_uff_E=324.01 & Chrysoeriol & -7.6 \\
\hline 9 & 4und_(1)_173183_uff_E=573.30 & Campesterol & -9.1 \\
\hline 10 & 4und_(1)_222284_uff_E=590.88 & Sitosterol & -8.8 \\
\hline 11 & 4und_(1)_5280805_uff_E=751.59 & Rutin & -8.9 \\
\hline
\end{tabular}

Table-3.

\begin{tabular}{|l|l|l|l|}
\hline Sr.No & Complex (protein + ligand) & Ligands & $\begin{array}{l}\text { Binding Affinity } \\
\text { (kcal/mol) }\end{array}$ \\
\hline 1 & 4und_(1)_247_uff_E=88.22 & betaine & -3.8 \\
\hline 2 & 4und_(1)_248_uff_E=88.90 & Trimethyl Glycine & -3.8 \\
\hline 3 & 4und_(1)_5280450_uff_E=147.99 & Linoleic acid & -5.9 \\
\hline 4 & 4und_(1)_445639_uff_E=80.35 & Oleic acid & -5.8 \\
\hline 5 & 4und_(1)_11005_uff_E=51.08 & myristic & -4.7 \\
\hline 6 & 4und_(1)_135191_uff_E=154.55 & xylose & -5.4 \\
\hline 7 & 4und_(1)_6137_uff_E=94.18 & methionine & -4.1 \\
\hline
\end{tabular}

Table- 4.

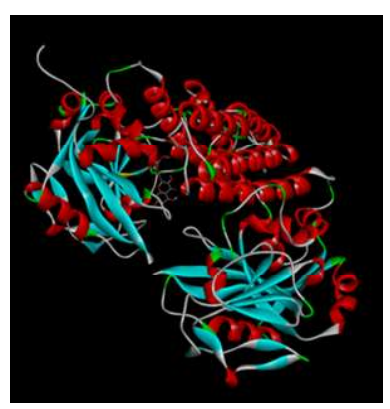

Silymarin

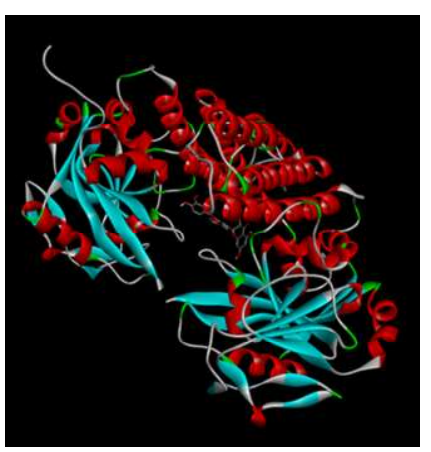

Silybinin

Fig. Binding of Chemical Constituents of Slybum Marianum with 4UND protein 


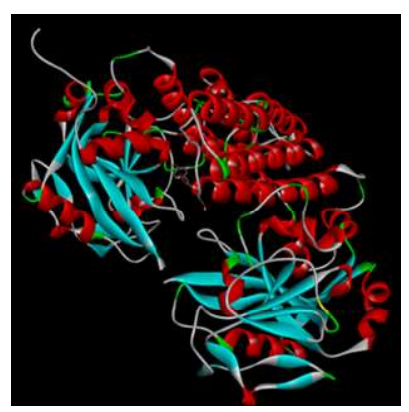

Dihydrokaempferol

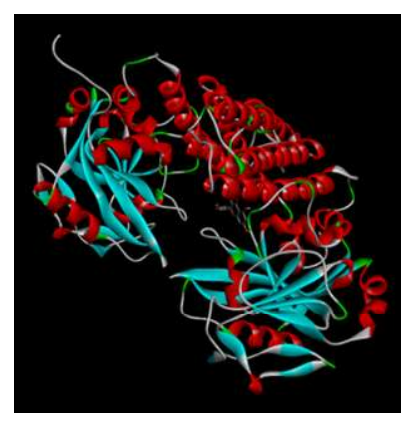

Kaempferol

Fig. Binding of Chemical Constituents of Slybum Marianum with 4UND protein

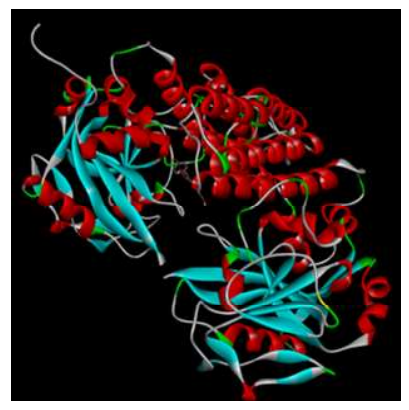

Dihydrokaempferol

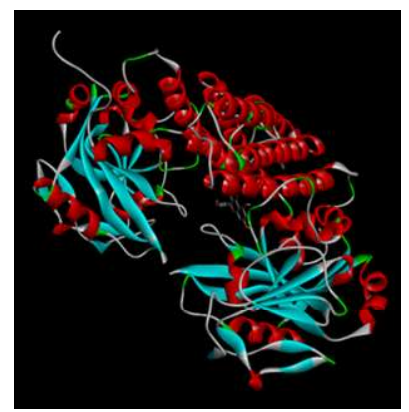

Kaempferol

Fig. Binding of Chemical Constituents of Slybum Marianum with 4UND protein 


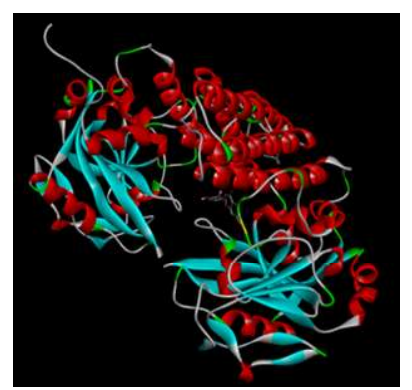

Eriodycitol

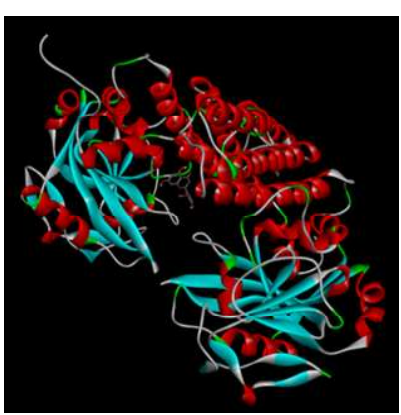

Chrysoeriol

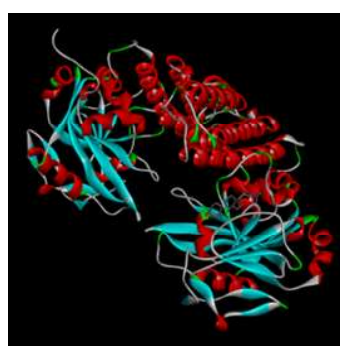

Campesterol

Fig. Binding of Chemical Constituents of Slybum Marianum with 4UND protein

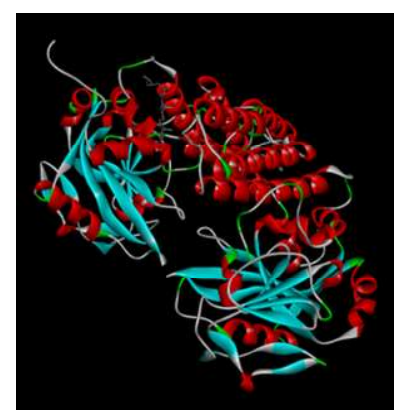

Sitosterol

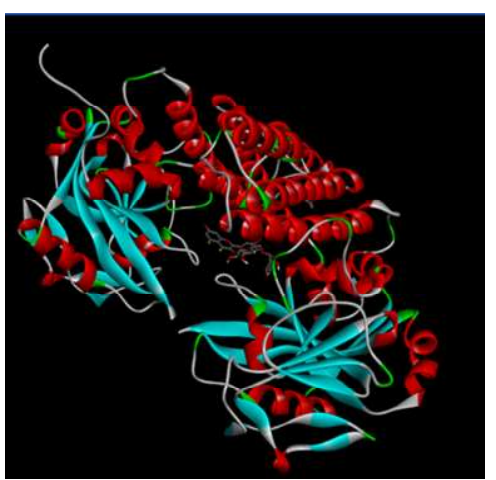

Rutin

Fig. Binding of Chemical Constituents of Slybum Marianum with 4UND protein 